\title{
Multi-Mode Antenna Enabled Direction-of-Arrival Estimation for Swarm Navigation
}

\author{
Robert Pöhlmann, Gonzalo Pedregosa, Stefano Caizzone, Emanuel Staudinger \\ German Aerospace Center (DLR) \\ Institute of Communications and Navigation \\ Oberpfaffenhofen, 82234 Wessling, Germany \\ Peter A. Hoeher \\ University of Kiel \\ Faculty of Engineering \\ 24143 Kiel, Germany \\ Email: \{Robert.Poehlmann, Gonzalo.Pedregosa, Stefano.Caizzone, Emanuel.Staudinger\}@dlr.de Email: ph@tf.uni-kiel.de
}

\begin{abstract}
Robotic swarm or multi-agent systems attract increasing attention for extraterrestrial exploration missions, among others. Control of a swarm requires communication among agents and accurate knowledge about their position and orientation. Both can be provided by radio signals exchanged between the agents. For navigation, round-trip delay (RTD) and direction-of-arrival (DoA) are informative signal metrics, as they relate to the relative distances and angles between the agents. DoA estimation is usually performed using antenna arrays. Recently, multi-mode antennas (MMAs) have been suggested as an alternative, but so far only theoretical results exist. This paper presents measurement results for DoA estimation with a single MMA mounted on a rover. The median estimation error of $7.2^{\circ}$ for a moving rover proves the potential of DoA estimation with an MMA.
\end{abstract}

\section{INTRODUCTION}

Autonomous robotic swarm systems, also called multi-agent systems, are considered for search and rescue [1], environmental monitoring [2], and extraterrestrial exploration missions [3], [4]. In comparison to a single robot, a swarm can explore a certain area faster, more reliable and from different perspectives, e.g. by ground-based and airborne agents. A swarm system brings inherent redundancy, failures of individual agents can be tolerated as long as enough functioning agents are left. For autonomous operation, where humans only interact with the swarm system by high-level commands, the swarm needs to be aware about its own state as well as its surroundings [5]. The swarm thus requires communication among agents and a reliable navigation solution.

On earth, global navigation satellite system (GNSS) is in general an option, but reception of the satellite signals could be blocked or impaired depending on the environment. For most extra-terrestrial missions, GNSS is not an option. Instead, signals directly exchanged within the agents of the swarm can be exploited. A combined swarm communication and navigation system has been investigated in [6]. The collaboration of agents for the purpose of positioning is called cooperative positioning. Fundamental limits for cooperative positioning can be found in [7]. Knowing only the position of the agents is in general not sufficient. For swarm control, e.g. to achieve a mission goal while keeping a favorable formation [8], [9], the orientation of the agents must be known as well. Fundamental limits for cooperative position and orientation estimation are investigated in [10], [11] for ideal antenna arrays and in [12] for multi-mode antennas (MMAs). A recent review regarding theoretical aspects of cooperative positioning can be found in [13], and regarding practical aspects in [14].

From a physical layer perspective, the basis for cooperative positioning is the extraction of position and orientation related metrics from the received radio signals. The time-of-arrival (ToA) is proportional to the distance among the agents [15], but it also contains their clock offset. For non-synchronized networks, the round-trip delay (RTD) can be measured instead [16]. Agent orientation is closely linked to the direction-ofarrival (DoA), which is classically observed using antenna arrays [17]. Recently, MMAs have been proposed as a new approach for DoA estimation [18]-[21]. An MMA is based on the theory of characteristic modes [22], [23] and can be defined as a multiport antenna, where different characteristic modes are excited independently. Examples for such antennas can be found in [24], [25]. DoA estimation with MMAs has so far been investigated theoretically and in simulations.

In this paper, we show measurement results for DoA estimation with a single MMA, which is integrated into a joint swarm communication and navigation system. First, we briefly introduce the physical layer of the system. We then provide theoretical background for DoA estimation with MMAs. The swarm communication and navigation system is implemented as a software-defined radio (SDR), which we proof to be suitable for DoA estimation by hardware-in-the-loop (HIL) simulation. As a main result, we present the DoA estimation performance with an MMA mounted on a rover which drives along a trajectory. Based on the measurement results, we conclude that an MMA is a suitable antenna for DoA estimation applications, specifically for swarm navigation.

\section{SWARM COMMUNICATION AND NAVIGATION SYSTEM}

Communication system requirements within a swarm, see Figure 1, are dictated by distributed algorithms forming the core of the swarm. Localization, exploration and control algorithms in general require broadcast communication with high update rates and low to medium sized packets [26]. The proposed system uses time-division multiple access (TDMA) 


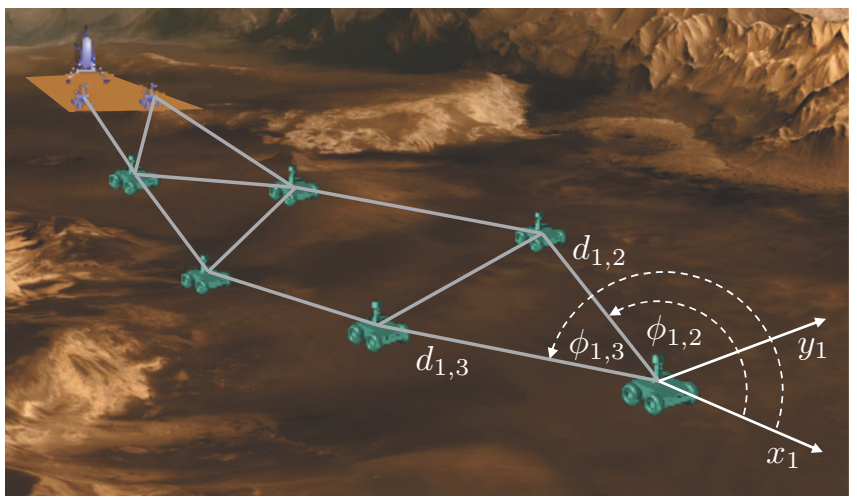

Fig. 1. Swarm with local frame of agent 1 and the ranges and DoAs with respect to its neighbors 2 and 3 .

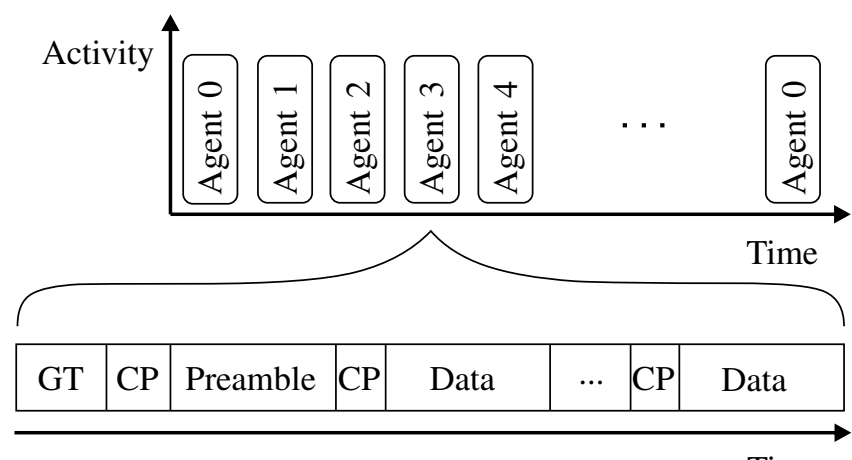

Time

Fig. 2. Self-organizing TDMA scheme and OFDM frame structure with TDMA guard time (GT) and cyclic prefix (CP).

for medium access control to avoid data packet collisions and ensure guaranteed update rates. In order to avoid a central point of failure, the TDMA scheme is designed in a selforganizing, decentralized manner. The employed modulation is orthogonal frequency-division multiplexing (OFDM) with 1024 subcarriers and a bandwidth of $25.6 \mathrm{MHz}$. Further parameters are detailed in Table I. The TDMA scheme as well as the packet structure is visualized in Figure 2. Each TDMA slot starts with a guard time, followed by multiple OFDM symbols, each with a cylic prefix. The first symbol is an agent-specific preamble which is used for synchronization and ToA estimation. The following symbols contain transmit timestamps and data to be exchanged within the swarm. The current system is designed for a maximum number of 20 agents, however system parameters are flexible and can be tailored to specific mission requirements and hardware constraints. Further details can be found in [26].

The relevant metrics to obtain knowledge about the agent positions and orientations within a swarm are distances and relative angles between the agents, see Figure 1. The distance can be obtained by measuring the ToA of radio signals. By encoding the transmission time, the time-of-flight (ToF) is calculated. The agents' clocks are not synchronized, thus twoway RTD ranging is employed to cancel out the clock offset. The estimated ToF is still impaired by drifting clocks during
TABLE I

SWARM COMMUNICATION AND NAVIGATION SYSTEM PARAMETERS.

\begin{tabular}{ll}
\hline Parameter & Value \\
\hline Carrier frequency & $1.68 \mathrm{GHz}$ \\
\hline Bandwidth & $25.6 \mathrm{MHz}$ \\
\hline Frame length $N$ & $1024 \mathrm{samples}$ \\
\hline Subcarrier spacing & $25 \mathrm{kHz}$ \\
\hline TDMA round-trip schedule & $100 \mathrm{~ms}$ \\
\hline Transmit power & $2 \mathrm{dBm}$ \\
\hline
\end{tabular}

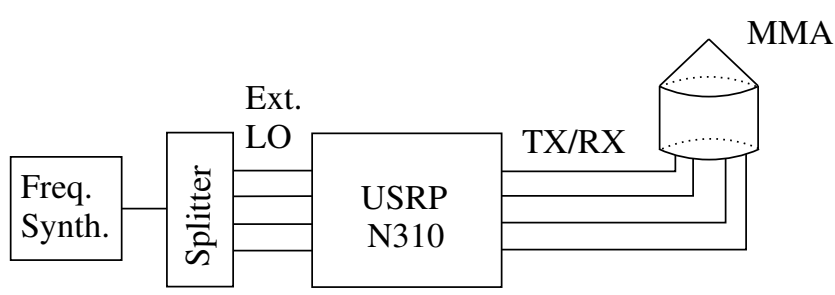

Fig. 3. Multichannel software-defined radio setup.

the two-way ranging process. To mitigate that, clock offsets and drifts are filtered using a clock model. A demonstrator of the envisioned system has been implemented using SDR, see [26].

The incident angle of a signal in the agent's local coordinate frame is called DoA. It can be observed by an MMA, which is detailed in the next section. A prerequisite is the extension of the system to allow DoA estimation with an MMA. This requires a phase-coherent multichannel receiver with phase and amplitude imbalances properly calibrated out. We use the Universal Software Radio Peripheral (USRP) N310 from Ettus Research, where we feed the local oscillator (LO) from an external frequency synthesizer, see Figure 3, to obtain phase coherent channels. Before operation, another frequency synthesizer is connected via a splitter to the TX/RX ports of the N310 to calibrate phase and amplitude imbalances between the channels. The receiver performance is verified by an HIL simulation in Section IV.

\section{DIRECTION-OF-ARRIVAL ESTIMATION WITH A MULTI-MODE ANTENNA}

An MMA is a multiport antenna, where each port features distinct radiation characteristics. Thus, an MMA with $M$ ports can be described by their gain pattern $g_{m}(\phi)$ and phase pattern $\Phi_{m}(\phi)$ [18], [27]. For port $m$ and DoA $\phi$ we get the antenna response

$$
a_{m}(\phi)=\sqrt{g_{m}(\phi)} e^{\mathrm{j} \Phi_{m}(\phi)} .
$$

The sampled baseband signal $\boldsymbol{r}(n)=\left[r_{1}(n), \ldots, r_{M}(n)\right]^{T}$ with sample index $n$ delivered by a calibrated multichannel receiver is defined as

$$
\boldsymbol{r}(n)=\boldsymbol{A}(\phi) \boldsymbol{s}(n)+\boldsymbol{w}(n),
$$

with the antenna response matrix

$$
\boldsymbol{A}(\boldsymbol{\phi})=\left[\begin{array}{lll}
\boldsymbol{a}\left(\phi_{1}\right) & \ldots & \boldsymbol{a}\left(\phi_{P}\right)
\end{array}\right]
$$


consisting of antenna response vectors

$$
\boldsymbol{a}(\phi)=\left[\begin{array}{lll}
a_{1}(\phi) & \ldots & a_{M}(\phi)
\end{array}\right]^{T},
$$

assuming that $P$ signals $s(n)=\left[s_{1}(n), \ldots, s_{P}(n)\right]^{T}$ arrive from DoAs $\phi_{1}, \ldots, \phi_{P}$. The signal bandwidth is assumed to be small compared to the carrier frequency [27], [28] and the system is internally noise limited, i.e. $\boldsymbol{w}(n) \sim \mathcal{C N}\left(0, \sigma_{w}^{2} \mathbb{I}_{M}\right)$ is independent and identically distributed (i.i.d.) white circular symmetric Gaussian noise. Following wavefield modeling and manifold separation, the antenna response vector

$$
\boldsymbol{a}(\phi)=\boldsymbol{G} \boldsymbol{b}(\phi)
$$

is decomposed into a product of the sampling matrix $G \in$ $\mathbb{C}^{M \times U}$, which is wavefield or DoA independent, and the basis vector $\boldsymbol{b}(\phi) \in \mathbb{C}^{U}$, which is antenna independent [29], [30].

The antenna response must be square integrable and the $U$ basis functions orthonormal on the manifold $\phi \in[-\pi, \pi)$. An extension to 3D is possible [18], [29], [30], but out of scope for this paper. A suitable basis for $2 \mathrm{D}$ is given by the Fourier functions

$$
\boldsymbol{b}(\phi)=\frac{1}{\sqrt{2 \pi}} e^{\mathrm{j} \phi u_{\phi}}, u_{\phi}=\left\lfloor-\frac{U-1}{2}\right\rfloor, \ldots, 0, \ldots,\left\lfloor\frac{U-1}{2}\right\rfloor .
$$

The order $U$ can be estimated by the electrical size of the antenna [29] or determined based on the measurement noise floor [31]. For the employed antenna we use $U=13$.

The MMA used in this stuy and developed at DLR [32] has been measured in an anechoic chamber at DoAs $\phi_{q}$ with $q=$ $1, . ., Q$, yielding $Q$ spatial samples of the antenna response for a specific DoA,

$$
\boldsymbol{e}_{q}=\left[\begin{array}{lll}
e_{q, 1} & \ldots & e_{q, M}
\end{array}\right]^{T}
$$

that can be joined to

$$
\boldsymbol{E}=\left[\begin{array}{lll}
e_{1} & \ldots & e_{Q}
\end{array}\right] .
$$

The sampling matrix $G$ is then obtained by least squares

$$
\boldsymbol{G}=\boldsymbol{E} \boldsymbol{B}^{H}\left(\boldsymbol{B} \boldsymbol{B}^{H}\right)^{-1},
$$

with $\boldsymbol{B}=\left[\begin{array}{lll}\boldsymbol{b}\left(\phi_{1}\right) & \ldots & \boldsymbol{b}\left(\phi_{Q}\right)\end{array}\right]$.

Figure 4 shows both the discrete antenna power and phase pattern $\boldsymbol{E}$ from the measurement chamber and the continuous antenna response vector $\boldsymbol{a}(\phi)$ obtained by wavefield modeling. Details about the employed antenna can be found in [32].

To estimate the DoA, we calculate the sample covariance matrix based on $N$ received signal samples

$$
\hat{\boldsymbol{R}}_{\boldsymbol{r}}=\frac{1}{N} \sum_{n=1}^{N} \boldsymbol{r}(n) \boldsymbol{r}^{H}(n)
$$

and evaluate the maximum likelihood (ML) estimator

$$
\hat{\boldsymbol{\phi}}=\arg \min _{\phi} \operatorname{Re}\left\{\operatorname{tr}\left\{\boldsymbol{\Pi}_{\boldsymbol{A}}^{\perp} \hat{\boldsymbol{R}}_{r}\right\}\right\}
$$

with $\Pi_{\boldsymbol{A}}^{\perp}=\mathbb{I}_{M}-\boldsymbol{A}(\phi) \boldsymbol{A}^{\dagger}(\phi)$, see e.g. [17], where $\dagger$ is the Moore-Penrose pseudoinverse.
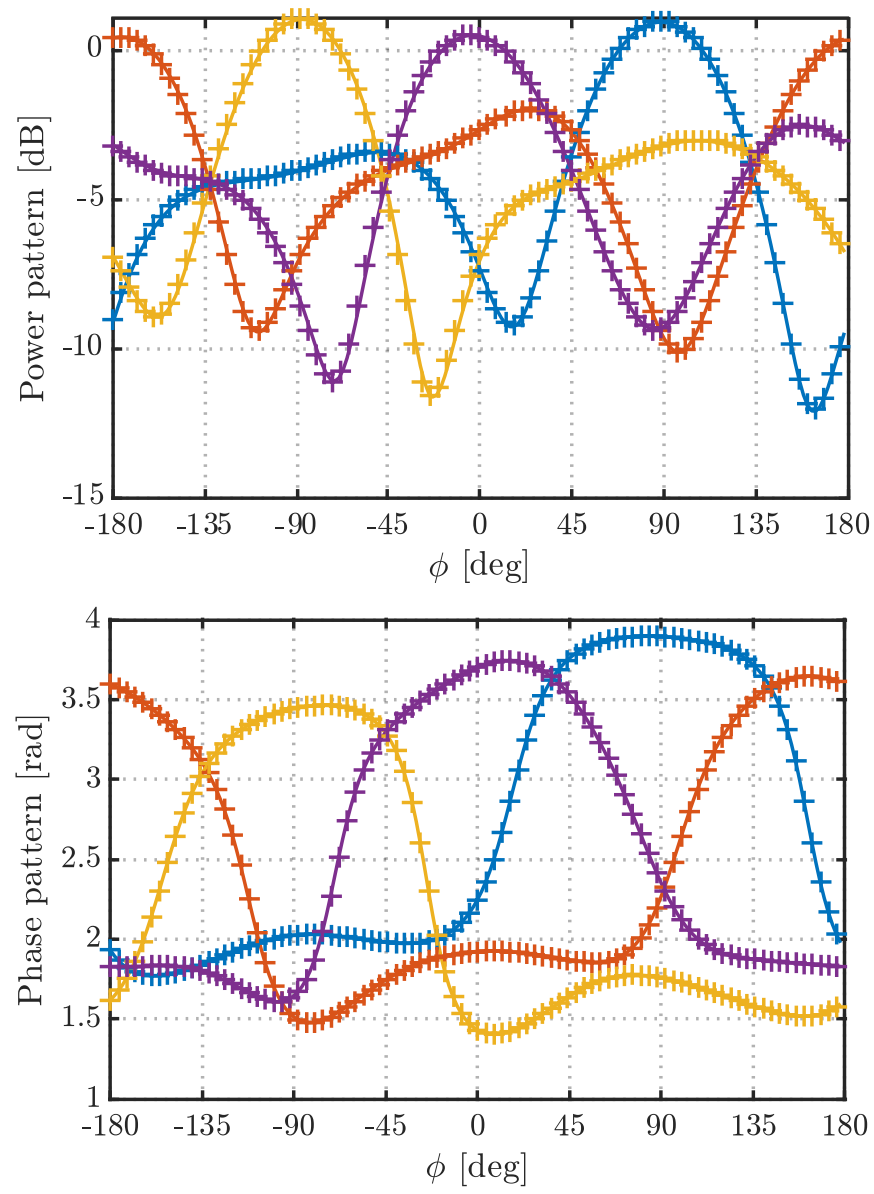

Port $1 \longleftarrow$ Port $2 \longleftarrow$ Port $3 \longleftarrow$ Port 4

Fig. 4. Discrete antenna power and phase pattern obtained in a measurement chamber together with continuous version from wavefield modeling.

\section{RESUlts}

\section{A. HIL simulation}

In order to verify the suitability of the SDR architecture for DoA estimation, we first perform an HIL simulation. Instead of the MMA, a transmitter is directly connected to the N310 with a four-way splitter. We receive a vector signal on four ports, $\boldsymbol{f}(s(n))$, where the function $\boldsymbol{f}($.$) represents$ both multiplicative receiver nonidealities like phase noise and amplitude variations and additive noise. Attenuation between transmitter and receiver was chosen to obtain a high signalto-noise ratio (SNR) of approx. $43 \mathrm{~dB}$, i.e. the additive noise in $\boldsymbol{f}(s(n))$ can be neglected as the SNR range of interest is $\ll 43 \mathrm{~dB}$. The received vector signal $\boldsymbol{f}(s(n))$ is stored and antenna response $\boldsymbol{A}(\phi)$ and noise $\boldsymbol{w}(n)$ are simulated. Based on (2), the HIL signal model is then

$$
\boldsymbol{r}(n)=\boldsymbol{A}(\phi) \cdot \boldsymbol{f}(s(n))+\boldsymbol{w}(n) .
$$

By this approach, receiver imperfections that are multiplicative w.r.t. the transmitted signal, e.g. phase noise and amplitude variations, are fully considered. It should be mentioned that it 


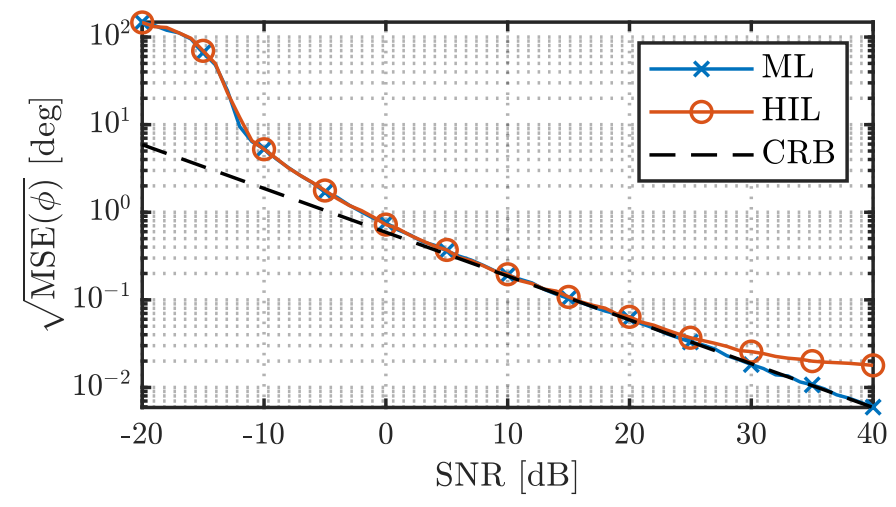

Fig. 5. DoA estimation RMSE at $\phi=50^{\circ}$ for ML simulation, HIL simulation and $\mathrm{CRB}$.

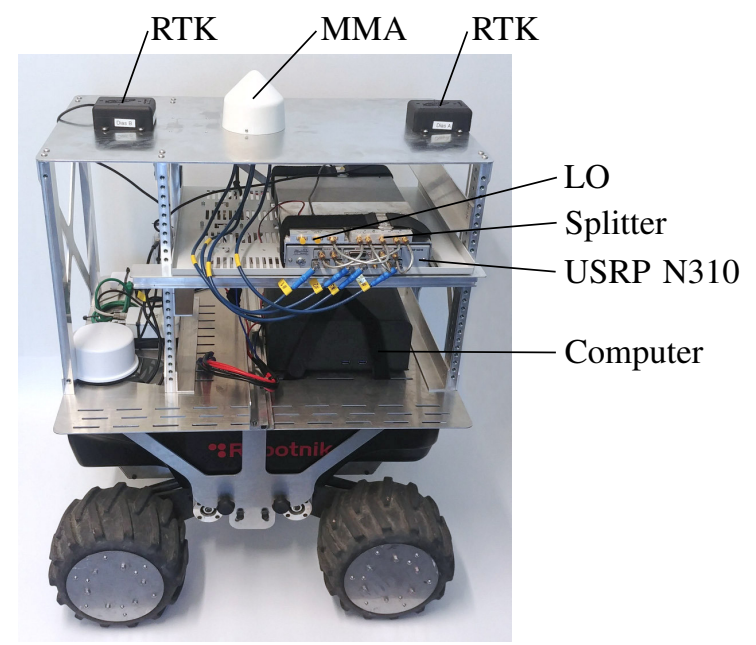

Fig. 6. Rover with installed multichannel SDR, see Figure 3, MMA, and two antenna RTK system for position and attitude reference [33].

is not possible to adjust the SNR in hardware by a tunable attenuator and then element-wise multiply the antenna response with the received vector signal, as this would lead to correlated noise and thus violate the signal model (2).

Figure 5 shows the root-mean-square error (RMSE) for varying SNR for a fixed DoA of $\phi=50^{\circ}$. The simulated ML estimator asymptotically approaches the Cramér-Rao bound (CRB), see e.g. [17], for high SNR. The HIL simulation is very close to the pure simulation, except for an SNR above $25 \mathrm{~dB}$, where the RMSE tends to flatten out. Apparently the influence of receiver phase noise and amplitude variations is small, as they average out when an OFDM frame of 1024 samples is considered. The receiver is thus well suited for DoA estimation.

\section{B. Outdoor experiment}

The multichannel SDR transceiver and MMA are mounted on a rover, see Figure 6, which can be used as a flexible experimental platform. The rover features a commercially available multi-sensor RTK system [33], which uses two GNSS RTK receivers, correction data from a nearby bases-

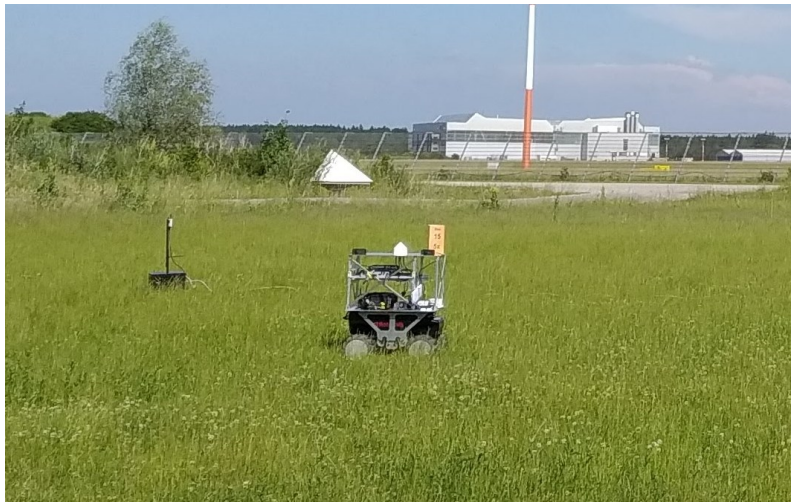

Fig. 7. Experiment environment with rover and static node.

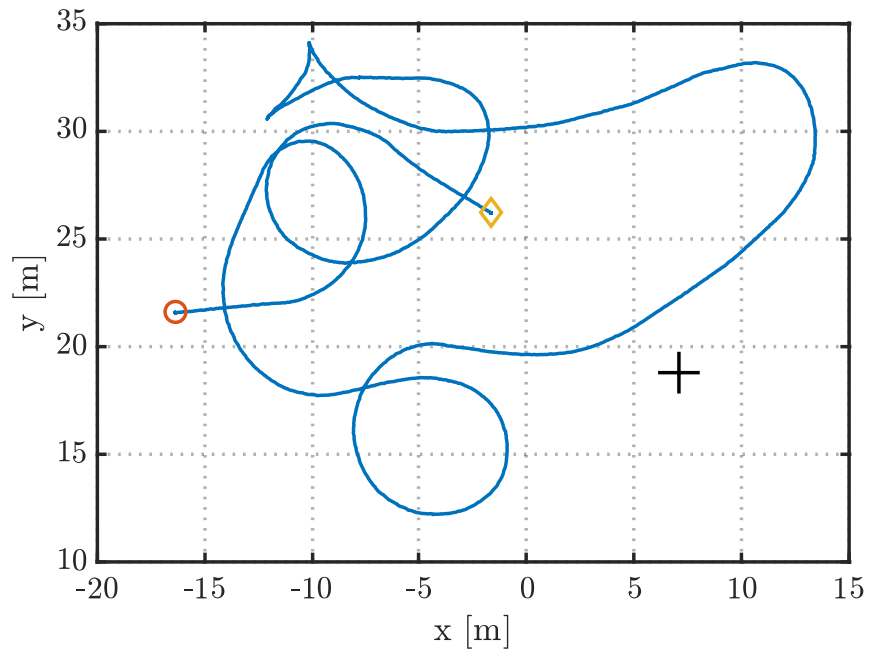

\begin{tabular}{lllll}
\hline Track & Start $\diamond$ & End & Anchor \\
\hline
\end{tabular}

Fig. 8. Map with track driven by the rover and a static anchor.

tation and inertial sensors. The obtained precise position and attitude solution is used as a reference.

The experiment was conducted in an outdoor environment with grassland, see Figure 7 . Figure 8 shows the track driven by the rover during a run of $9 \mathrm{~min} 27 \mathrm{~s}$ and the anchor position. Except for a short section starting at $x=-10 \mathrm{~m}, y=35 \mathrm{~m}$, the rover was driving forward. In Figure 9, reference and estimated DoAs are shown over the traveled time. The reference DoA is calculated from the reference position and attitude of the rover and the known anchor position. To allow better visual assessment, the $360^{\circ}$ jumps are unwrapped. According to the plot, the estimation is close to the true value, except for a few outliers. In Figure 10, the estimation error is shown over time. The median estimation error is $7.2^{\circ}$. For short periods, burstlike errors up to $95^{\circ}$ occur. These large outliers can in practice be detected and discarded by a subsequent positioning filter, which may also use other sensors, e.g. inertial or optical. The rover was driving on bumpy grassland which caused the rover to wobble during the movement. Both shaking and the uneven surface result in roll and pitch angles of the rover not being 


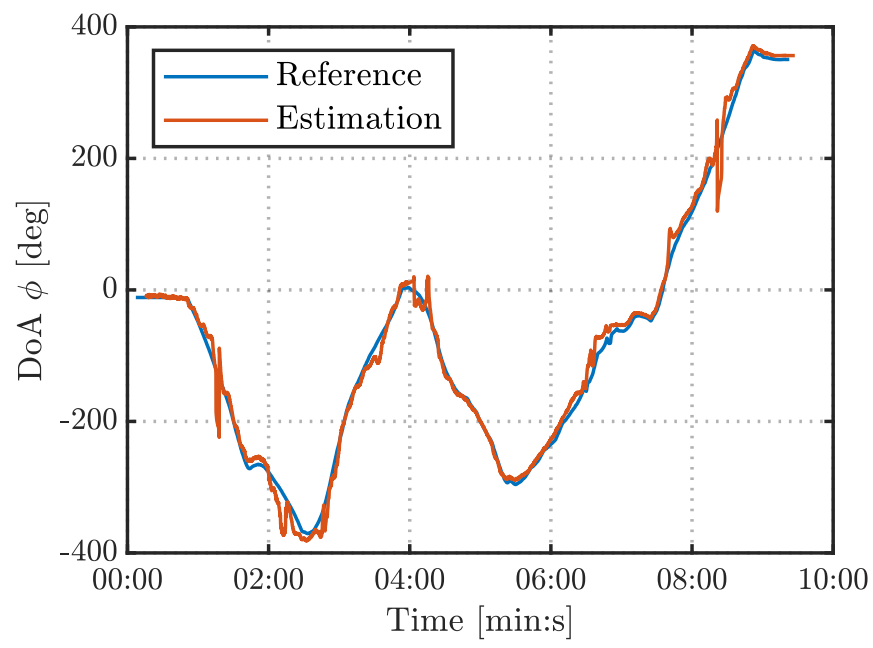

Fig. 9. DoA reference and estimation for the rover traveling time of $9 \mathrm{~min}$ $27 \mathrm{~s}$. The $360^{\circ}$ jumps are unwrapped for this plot.

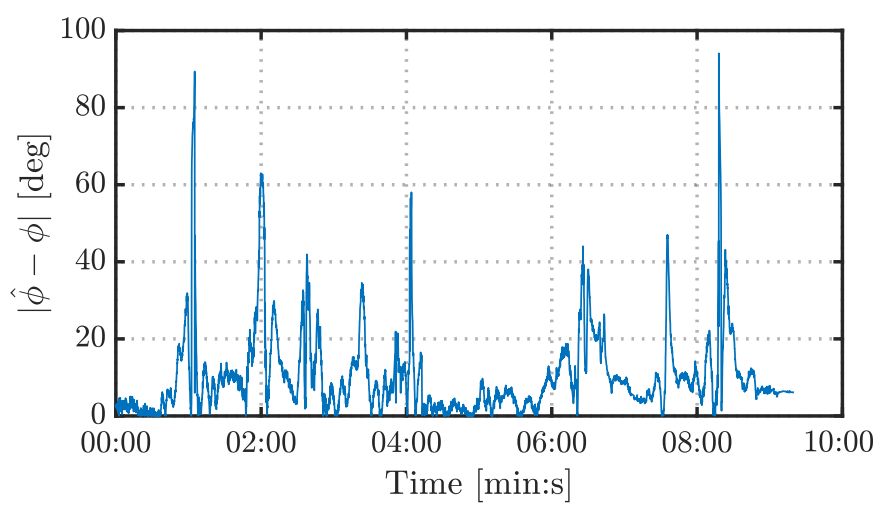

Fig. 10. Estimation error for the rover traveling time of $9 \mathrm{~min} 27 \mathrm{~s}$.

exactly zero. This causes a model mismatch for the antenna pattern, as the signal is in this case not arriving exactly at the equator plane of the antenna, and could explain the estimation errors. According to the empirical CDF in Figure 11, the error was below $14.6^{\circ}$ for $80 \%$ of the time and below $22.8^{\circ}$ for $90 \%$ of the time. It should be mentioned that although we show results for a single link, the system can be operated with multiple agents.

\section{CONClusion}

In this paper, we present first measurement results for DoA estimation with an MMA, a topic which has so far been treated only theoretically. A coherent multichannel SDR is developed and integrated into a swarm communication and navigation system. Its suitability for DoA estimation is shown with an HIL simulation. SDR and MMA are mounted on a rover, which dynamically drives around an area and communicates with a static node. Despite the uneven ground, resulting in slight misalignment and shaking of the rover, the median DoA estimation error is $7.2^{\circ}$. According to the present results, DoA estimation using a single MMA appears feasible. Further

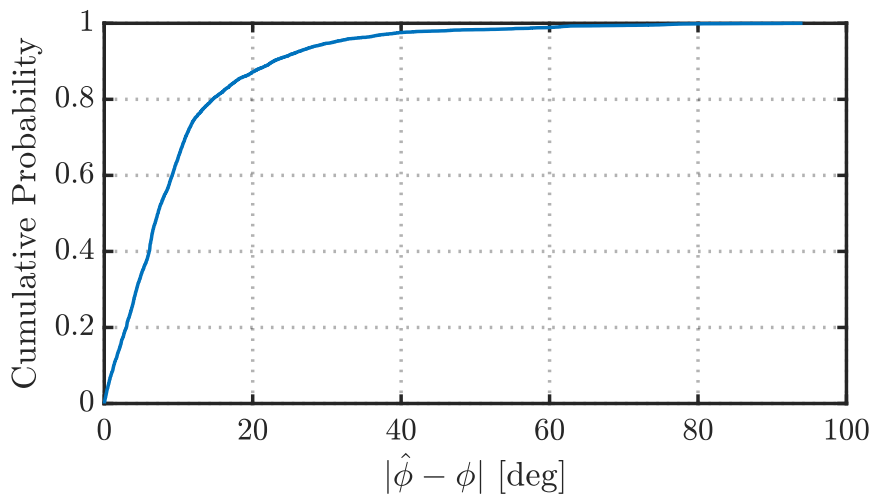

Fig. 11. Empirical CDF of the estimation error.

improvements to obtain a lower estimation error are currently being investigated.

\section{ACKNOWLEDGMENT}

This work has been funded by the German Research Foundation (DFG) under contract numbers FI 2176/1-2 and HO 2226/17-2.

\section{REFERENCES}

[1] M. Bernard, K. Kondak, I. Maza, and A. Ollero, "Autonomous transportation and deployment with aerial robots for search and rescue missions," Journal of Field Robotics, vol. 28, no. 6, pp. 914-931, 2011.

[2] M. Dunbabin and L. Marques, "Robots for environmental monitoring: Significant advancements and applications," IEEE Robotics Automation Magazine, vol. 19, no. 1, pp. 24-39, Mar. 2012.

[3] A. Seeni, B. Schfer, and G. Hirzinger, "Robot mobility systems for planetary surface exploration - state-of-the-art and future outlook: A literature survey," in Aerospace Technologies Advancements, T. T., Ed. London: InTech, Jan. 2010, pp. 189-208.

[4] A. Wedler, M. Wilde, A. Dömel, M. G. Müller, J. Reill, M. Schuster, W. Stürzl, R. Triebel, H. Gmeiner, B. Vodermayer, Bussmann, M. Vayugundla, S. Brunner, H. Lehner, P. Lehner, A. Börner, R. Krenn, A. Dammann, U.-C. Fiebig, E. Staudinger, F. Wenzhöfer, S. Flögel, S. Sommer, T. Asfour, M. Flad, S. Hohmann, M. Brandauer, and A. O. Albu-Schäffer, "From single autonomous robots toward cooperative robotic interactions for future planetary exploration missions," in Proc. Int. Astronautical Congr., IAC, Bremen, Germany, Oct. 2018.

[5] W. Truszkowski, M. Hinchey, J. Rash, and C. Rouff, "NASA's swarm missions: The challenge of building autonomous software," IT Professional, vol. 6, no. 5, pp. 47-52, Sep. 2004.

[6] S. Zhang, E. Staudinger, S. Sand, R. Raulefs, and A. Dammann, "Anchor-free localization using round-trip delay measurements for martian swarm exploration," in Proc. IEEE/ION Position, Location and Navigation Symp. - PLANS 2014, May 2014, pp. 1130-1139.

[7] Y. Shen, H. Wymeersch, and M. Z. Win, "Fundamental limits of wideband localization-Part II: Cooperative networks," IEEE Transactions on Information Theory, vol. 56, no. 10, pp. 4981-5000, Oct. 2010.

[8] K.-K. Oh, M.-C. Park, and H.-S. Ahn, "A survey of multi-agent formation control," Automatica, vol. 53, pp. 424-440, Mar. 2015.

[9] S. Zhang, R. Raulefs, and A. Dammann, "Location information driven formation control for swarm return-to-base application," in Proc. 24th European Signal Processing Conf. (EUSIPCO), Aug. 2016, pp. 758763.

[10] Y. Shen and M. Win, "On the accuracy of localization systems using wideband antenna arrays," IEEE Transactions on Communications, vol. 58, no. 1, pp. 270-280, Jan. 2010.

[11] R. Pöhlmann, S. Zhang, A. Dammann, and P. A. Hoeher, "Fundamental limits for joint relative position and orientation estimation," in Proc. IEEE Int. Conf. Communications Workshops (ICC Workshops), Kansas City, Missouri, May 2018. 
[12] — , "Fundamental limits for joint relative position and orientation estimation with generic antennas," in Proc. 26th European Signal Processing Conf. (EUSIPCO), Rome, Italy, 2018, pp. 697-701.

[13] M. Z. Win, Y. Shen, and W. Dai, "A theoretical foundation of network localization and navigation," Proceedings of the IEEE, vol. 106, no. 7, pp. 1136-1165, Jul. 2018.

[14] R. M. Buehrer, H. Wymeersch, and R. M. Vaghefi, "Collaborative sensor network localization: Algorithms and practical issues," Proceedings of the IEEE, vol. 106, no. 6, pp. 1089-1114, Jun. 2018.

[15] D. Dardari, A. Conti, U. Ferner, A. Giorgetti, and M. Z. Win, "Ranging with ultrawide bandwidth signals in multipath environments," Proceedings of the IEEE, vol. 97, no. 2, pp. 404-426, Feb. 2009.

[16] E. Staudinger, S. Zhang, and A. Dammann, "Cramér-Rao lower-bound for round-trip delay ranging with subcarrier-interleaved OFDMA," IEEE Transactions on Aerospace and Electronic Systems, vol. 52, pp. 29612972, Dec. 2016.

[17] M. Viberg, "Introduction to array processing," in Array and Statistical Signal Processing, ser. Academic Press Library in Signal Processing, A. M. Zoubir, M. Viberg, R. Chellappa, and S. Theodoridis, Eds. Boston: Elsevier, 2014, vol. 3, ch. 11, pp. 463-502.

[18] R. Pöhlmann, S. A. Almasri, S. Zhang, T. Jost, A. Dammann, and P. A. Hoeher, "On the potential of multi-mode antennas for direction-of-arrival estimation," IEEE Transactions on Antennas and Propagation, vol. 67, no. 5, pp. 3374-3386, May 2019.

[19] S. A. Almasri, R. Pöhlmann, N. Doose, P. A. Hoeher, and A. Dammann, "Modeling aspects of planar multi-mode antennas for direction-of-arrival estimation," IEEE Sensors Journal, vol. 19, no. 12, pp. 4585-4597, Jun. 2019.

[20] R. Pöhlmann, S. Zhang, T. Jost, and A. Dammann, "Power-based direction-of-arrival estimation using a single multi-mode antenna," in Proc. 14th Workshop Positioning, Navigation and Communications (WPNC), Bremen, Germany, Oct. 2017.

[21] S. A. Almasri, N. Doose, and P. A. Hoeher, "Parametric direction-ofarrival estimation for multi-mode antennas," in Proc. 14th Workshop Positioning, Navigation and Communications (WPNC), Bremen, Germany, Oct. 2017.

[22] R. Garbacz and R. Turpin, "A generalized expansion for radiated and scattered fields," IEEE Transactions on Antennas and Propagation, vol. 19, no. 3, pp. 348-358, May 1971.

[23] R. Harrington and J. Mautz, "Theory of characteristic modes for conducting bodies," IEEE Transactions on Antennas and Propagation, vol. 19 , no. 5, pp. 622-628, Sep. 1971.

[24] D. Manteuffel and R. Martens, "Compact multimode multielement antenna for indoor UWB massive MIMO," IEEE Transactions on Antennas and Propagation, vol. 64, no. 7, pp. 2689-2697, Jul. 2016.

[25] E. Antonino-Daviu, M. Cabedo-Fabres, M. Gallo, M. Ferrando-Bataller, and M. Bozzetti, "Design of a multimode MIMO antenna using characteristic modes," in Proc. 3rd European Conf. Antennas and Propagation, Mar. 2009, pp. 1840-1844.

[26] E. Staudinger, D. Shutin, C. Manss, A. Viseras, and S. Zhang, "Swarm technologies for future space exploration missions," in Proc. I-SAIRAS '18: Int. Symp. Artificial Intelligence, Robotics and Automation in Space, Madrid, Spanien, Jun. 2018.

[27] C. A. Balanis and P. I. Ioannides, Introduction to Smart Antennas. San Rafael: Morgan \& Claypool Publishers, 2007.

[28] M. Viberg and A. Zoubir, Array and Statistical Signal Processing, ser. Academic Press Library in Signal Processing. Boston: Academic Press, 2014.

[29] M. A. Doron and E. Doron, "Wavefield modeling and array processing. I. Spatial sampling," IEEE Transactions on Signal Processing, vol. 42, no. 10 , pp. 2549-2559, 1994

[30] M. Costa, A. Richter, and V. Koivunen, "Unified array manifold decomposition based on spherical harmonics and 2-D fourier basis," IEEE Transactions on Signal Processing, vol. 58, no. 9, pp. 4634-4645, Sep. 2010.

[31] F. Belloni, A. Richter, and V. Koivunen, "DoA estimation via manifold separation for arbitrary array structures," IEEE Transactions on Signal Processing, vol. 55, no. 10, pp. 4800-4810, Oct. 2007.

[32] S. Caizzone, M. S. Circiu, W. Elmarissi, and C. Enneking, "All-GNSSband DRA antenna for high-precision applications," in Proc. 12th European Conf. Antennas and Propagation (EuCAP 2018), Apr. 2018, pp. 543-547.

[33] “ANAVS Multi-Sensor RTK Module," https://www.anavs.de. 